\title{
A Method For The Verification Of Wire Crimp Compression Using Ultrasonic Inspection
}

K. E. Cramer, D. F. Perey and W. T. Yost

NASA Langley Research Center, Hampton, VA

\begin{abstract}
The development of a new ultrasonic measurement technique to assess quantitatively wire crimp terminations is discussed. The amplitude change of a compressional ultrasonic wave propagating at right angles to the wire axis and through the junction of a crimp termination is shown to correlate with the results of a destructive pull test, which is a standard for assessing crimp wire junction quality. To demonstrate the technique, the case of incomplete compression of crimped connections is ultrasonically tested, and the results are correlated with pull tests. Results show that the nondestructive ultrasonic measurement technique consistently predicts good crimps when the ultrasonic transmission is above a certain threshold amplitude level. A quantitative measure of the quality of the crimped connection based on the ultrasonic energy transmitted is shown to respond accurately to crimp quality. A wave propagation model, solved by finite element analysis, describes the compressional ultrasonic wave propagation through the junction during the crimping process. This model is in agreement within $6 \%$ of the ultrasonic measurements. A prototype instrument for applying this technique while wire crimps are installed is also presented. The instrument is based on a two-jaw type crimp tool suitable for butt-splice type connections. A comparison of the results of two different instruments is presented and shows reproducibility between instruments within a $95 \%$ confidence bound.
\end{abstract}




\section{Introduction}

A wire-crimp terminator is a fixture which consists of a mating end and a crimp ferrule, and is installed on the end of a wire. This establishes a secure mechanical and electrical connection between the wire and the terminator. A terminator is installed onto the end of a wire by removing a portion of the insulation to expose a prescribed length of wire strands, and placing them inside the ferrule. The ferrule is deformed by a tool designed to force the wire surfaces and the ferrule's inner surface into intimate contact. This connection then maintains a residual force by the ferrule on the wire to assure good mechanical and electrical continuity between the wire's end and the terminator.

In critical applications specialized tools are used for optimum crimp quality. Procedures are developed and representative crimps are formed using the prescribed method. To ensure quality the crimps are checked periodically by destructively pulling to failure (recording the pull load at failure) or sectioning for microscopic inspection. In critical work, such as space shuttle wiring, the tools are checked before and after each application. The checkout procedure for a specialized tool may include forming representative crimps that are pull-tested to failure or using gauge pins to verify proper tool die compression. A tool passing these tests is certified for the next use. A crimp formed in the field using the prescribed procedures with a certified tool is assumed to be of good quality. However, other than visual inspection and a cursory electrical continuity check, the critical crimp is generally not independently verified for quality.

Crimps fail for many reasons. Installation-related failures include failure to match terminator choice to wire size to crimp tool, improper crimping technique, failure to fully insert the wire into the terminator, and improper removal of the insulation. Further, if the crimp tool has structural damage (e.g. worn or damaged joints or jaws), the mechanical reliability of the 
connection can be compromised.

Present practices for assuring good crimps are based on the practitioner following detailed crimping procedures, with quality assurance verifications at each step. This approach assures a good initial crimp, assuming that the connector integrity is good. However, no independent means to test and confirm each and every crimp is currently available. This work presents a new and novel way to verify nondestructively the quality of all crimps by examining ultrasonic transmission through the crimp, at the time that it is formed. A prototype crimp tool is developed to include the ultrasonic transmission apparatus. Ultrasound provides the means of assessing crimp quality that ensures the electrical and mechanical integrity of an initial crimp before the installation process is completed.

\section{Ultrasonic Based Inspection Technique}

The purpose of a well-crimped junction is to provide both good electrical and mechanical coupling of wire to the ferrule. This condition is achieved by creating sufficient residual stresses between the wire strand surfaces and the terminator by deforming the ferrule around the wire. These deformation-induced stresses hold the wire in intimate contact with the ferrule. If these stresses relax or change, the intimate contact is not assured, and the crimped connection may fail.

The technique presented here uses ultrasonic wave transmission through the ferrule-wire joint (perpendicular to the geometric axis) to inspect a mechanically crimped connection. Figure 1 shows a schematic of the basic components. The transmit transducer generates an ultrasonic wave, which travels into the jaw of the crimp tool. Upon application of pressure, the jaw deforms the crimp ferrule around the wire with sufficient force to press the wire and ferrule surfaces into intimate mechanical contact. This allows transmission of the ultrasonic wave through the 
ferrule, through the electrical wire, and into the opposite jaw to be received by an ultrasonic transducer. As the pressure increases, conformation between the crimp tool jaws, the ferrule, and the enclosed wire strands improves, and more of the ultrasonic wave energy propagates through the ferrule-wire joint.

The transducer configuration on the crimp tool requires that the ultrasonic wave propagates through the crimp and wire. If insufficient contact exists between the wires and ferrule surface, or between the wire strands themselves, then the resulting signal is low. On the other hand, if the crimp produces sufficient contact between the wire stands and the ferrule, ultrasonic transmission is high. A necessary condition for an ultrasonic wave to traverse the wire strandferrule joint is that the wire strands and ferrule must be in intimate contact at the atomic levels. For this analysis we choose to examine the first received pulse (FRP) transmission through the crimp.

The experimental setup consists of an ultrasonic pulser-receiver (Panametrics Model 5900PR) in a pitch-catch arrangement as shown in Figure 1. The send and receive transducers are damped PZT-5 ultrasonic compressional wave transducers, $7.5 \mathrm{MHz}$ center frequency, $6.35 \mathrm{~mm}$ diameter (Panametrics Model A121S). The crimp tool (Raychem Model 80-1377) is a commercially available unit that was modified by adding wedges to provide parallel send and receive planes for mounting the transducers[1,2]. Only the center crimp die was instrumented for this test. Figure 2 shows a laboratory photograph of the instrumented crimp tool.

For this study 16-gauge (MIL 22759/34-16-9) wire size was selected. Sixteen-gauge wire consists of 19 individual strands of 29 -gauge nickel plated copper (.29 mm diameter each). The insulation is stripped and inserted into a nickel plated, copper ferrule (MIL M39029/31-228). 
The ferrule has a $2.7 \mathrm{~mm}$ outside diameter and $1.6 \mathrm{~mm}$ inside diameter. This terminator is used when making butt-splice type connections. The uncrimped bundle diameter inside the insulation is $1.37 \mathrm{~mm}$. The ferrule end of the terminator is inserted on the wire, and then the loaded ferrule is placed in the slot of the jaws of the instrumented crimp tool. The ultrasonic pulser-receiver is connected to the appropriate transducers, and the crimp tool is compressed while the ultrasonic sensors are active.

\section{Model of Ultrasonic Wave Propagation Through a Crimped Connector}

An ultrasonic wave model for propagation of ultrasound through a crimped electrical connection was developed to confirm the assumption that the FRP consisted of a compressional wave that passes directly through the connector (with no reflections or mode conversions). While this model contains the transmission of both compressional and shears waves, the analysis confirms that only the compressional wave mode unambiguously explains the FRP.

Figure 3 shows a cross-sectional schematic of a wire-ferrule segment, the crimp tool jaws, and the ultrasonic transducers. For a 16-gauge ferrule the nominal outside diameter is $2.7 \mathrm{~mm}$ and the material is nickel-plated copper. The crimp tool is steel and the total distance between transducers is $35.5 \mathrm{~mm}$. The compressional velocity of sound $\left(\mathrm{V}_{\mathrm{L}}\right)$ in copper is $5.01 \mathrm{x} 10^{6} \mathrm{~mm} / \mathrm{s}$ and the shear wave velocity $\left(\mathrm{V}_{\mathrm{S}}\right)$ is $2.27 \times 10^{6} \mathrm{~mm} / \mathrm{s}$. Likewise for steel $\mathrm{V}_{\mathrm{L}}$ is $5.9 \times 10^{6} \mathrm{~mm} / \mathrm{s}$ and $\mathrm{V}_{\mathrm{S}}$ is $3.2 \times 10^{6} \mathrm{~mm} / \mathrm{s}[3]$. The effect of the nickel plating on the ultrasonic propagation is neglected. For the geometry of Figure 3, the total propagation time from the transmit to the receive transducer for a compressional wave is $6.55 \mu \mathrm{s}$. Shear waves created by mode conversion at the copper-to-steel interface closest to the receiving transducer would arrive, at the earliest, $2.54 \mu$ s later. Since these transducers are sensitive to compressional waves, a second possibility exists where a compressional wave is mode converted to a shear wave at interface A, 
then mode converted back to a compressional wave at interface B. In this case the total propagation time would be at $7.14 \mu \mathrm{s}$. Therefore, if the analysis of the received data is limited to a time window of 6.0 to $7.0 \mu$ s, only compressional waves will be present and need to be considered in the model.

Once the crimp is compressed, this timing will shift slightly due to shortening of the total path length. This time shift is small due to the compression occurring in the copper connector and the consequent rearrangement of the copper strands in the wire (thus minimizing free space between the strands in the crimp area). If the outside diameter of the ferrule-wire portion of the connector is reduced from $2.7 \mathrm{~mm}$ to $1.35 \mathrm{~mm}$ (resulting in 50\% reduction in signal path length, typical for a fully compressed connection), the time shift is $0.27 \mu \mathrm{s}$, which is within the width of the time window selected. Experimental results, shown in Figure 4, confirm that this is the first signal to arrive at the receiving transducer.

Finally, we consider a reflected compressional wave after its propagation through the crimp connector (Figure 3) and its reflection at interface C. Suppose that it reflects again at interface B into the receive transducer. The propagation time for this sequence is $13.15 \mu \mathrm{s}$. This is again outside the time window of interest.

The summary of the above analyses confirms that the first arrival signal at the receive transducer occurs at approximately $6.5 \mu$ s and can therefore be modeled as compressional waves only.

\section{Data Analysis}

Consistent with the width of the FRP shown above, a time window of $1.0 \mu$ s, centered on the FRP, was selected for analysis. The data analysis consists of performing a Hilbert transform[4] on the ultrasonic signal. The modulus of the transform is integrated (summed) over the time 
window:

$\eta=\sum_{t_{1}}^{t_{2}} H H^{*}$

Where $t_{1}$ and $t_{2}$ are the starting and ending times of the time window and $\mathrm{H}$ is the Hilbert transform of the ultrasonic signal. This results in a value $(\eta)$ proportional to the ultrasonic energy transmitted through the crimp[5]. These values are plotted against the load at failure for each specimen.

\section{Ultrasonic Transmission Measurements for Various Pathologies}

\subsection{Incomplete Compressions}

Data are acquired on a series of 16-gauge wire crimps with varying degrees of under-crimp ferrule-wire compression. The opening distance of the crimp tool jaws, as referenced by the distance between the two handles, is used as a measure of the amount of compression.

Ultrasonic transmission data are collected after compressing the jaws to a prescribed distance. The jaws are then released, and the wire/crimp is removed. The wire/crimp is mounted on the specimen stage of a wire crimp pull tester (Alphatron Model MPT-200A) and mechanically pulled to failure. The failure load is recorded.

Figure 5 shows the results for the 96 specimens. These are divided into 6 data runs of 16 partial to full crimps each. The data are plotted on a log-log scale, shown in Figure 5. The horizontal 
line drawn on the graph for reference is the load above which a 16-gauge crimp is deemed acceptable (50 lbs per SAE-AS7928/1 specification). The value of $\eta$ correlates well with the failure load data for these under-crimped terminators. If one were to select a value $(\eta)$ of approximately 150 , then a $97 \%$ detection rate (93/96) and a $1 \%$ false call rate $(1 / 96)$ would result. Only a few data points near $\eta=10^{5}$ (full horizontal scale) actually represent fully compressed crimps where the tool detent prawl would ordinarily release to allow crimp removal. This suggests that many under crimped specimens would pass the $50 \mathrm{lbs}$ standard load test.

\subsection{Instrumentation Reproducibility}

To demonstrate reproducibility of the technique, independent of the hardware used, a second crimp tool is instrumented identically to the first tool. A series of under-crimped connectors are measured with a second tool and the transmitted ultrasonic signal is recorded. These connectors are pulled to failure using the procedure described previously. A comparison of the ultrasonic responses for the two tools is presented in Figure 6. The solid line on is a least squares curve fit of the $\log$ of failure load as a function of the log of the crimp attenuation factor for the first tool. The dashed line represents the 95\% confidence bound of the least squares fit for the first tool. All but the smallest $\eta$ values from the second tool fit within the 95\% confidence bound of the first tool.

\section{X-ray Computed Tomography of Electrical Crimp}

To assist in developing an understanding of the sound propagation in an electrical crimp, x-ray computed tomography $(\mathrm{CT})$ is performed on three 16-gauge wire crimps with varying levels of ferrule compression. The CT system is a custom microfocus system with $25 \mu \mathrm{m}$ spot size and $12.5 \mu \mathrm{m}$ resolution. Figure 7 shows a photograph of a fully crimped connector and eight CT slices across that connector, demonstrating the amount of compression achieved. In addition to a 
fully compressed crimp, one is also produced at a very small amount of compression and one at approximately $50 \%$ of full compression. As before, crimp compression is measured by closure of the crimp tool handle: $5 \mathrm{~mm}$ closure corresponds to very little compression, $20 \mathrm{~mm}$ corresponds to approximately $50 \%$ of full compression and $32.5 \mathrm{~mm}$ corresponds to full compression. The CT slices in Figure 7 show regions of linear porosity (empty or "free" volume) that decrease with increasing levels of crimp compression. Changes in ultrasonic attenuation have previously been used to measure porosity in composite materials[6-9] and in cast metals[10,11].

Figure 8 shows a graph of the ultrasonic signal generated at each compression level. For comparison a single CT slice across the region with the largest compression is provided for each crimp. The amplitude of the FRP increases with an increasing level of crimp compression. This supports the relationship between ferrule-wire compression and ultrasonic transmission mentioned previously.

\section{Finite Element Model for Ultrasonic Transmission Through Ferrule-Wire Compression Region}

A finite element model (FEM) was developed using the commercial software COMSOL Multiphysics ${ }^{\circledR}$. The two-dimensional, time-dependent model, shown in Figure 9, consisted of 9000 elements and 19507 degrees of freedom. The jaw of the crimp tool is made from steel (compressional wave speed is $5.9 \times 10^{3} \mathrm{~m} / \mathrm{s}$ and the density is $7.9 \times 10^{3} \mathrm{~kg} / \mathrm{m}^{3}$ ); the crimp ferrule and wire are copper (compressional wave speed is $5.0 \times 10^{3} \mathrm{~m} / \mathrm{s}$ and the density is $8.9 \times 10^{3}$ $\left.\mathrm{kg} / \mathrm{m}^{3}\right)[4]$. The ultrasonic excitation is a $100 \mathrm{~ns}$ wide square pulse of unit amplitude.

Figure 10 shows a comparison of the experimental results and the FEM of the crimped connector. To minimize the effects of transducer response, which was not included in the model, 
the square of the modulus of the Hilbert Transform of each signal is shown in Figure 10. When integrated over a $1 \mu$ s time window $(6.25-7.25 \mu \mathrm{s})$, the model agrees with the experiment to within $6 \%\left(\eta_{\text {model }}=425.5\right.$ and $\eta_{\text {experiment }}=451.03$ (normalized units $\left.)\right)$.

\section{Conclusions}

We report here a new measurement technique to assess quantitatively the quality of wire crimp terminations during terminator installation. Because the timing of the received pulses remains nearly constant for all test conditions, with only the amplitude changing, a Hilbert Transform calculation (square-root of the Hilbert Transform modulus) of the received transmission ultrasonic waveform data with integration over a fixed time window has been shown in this paper to produce a reproducible, quantitative measure of crimp quality for the cases examined. A finite element model of wave propagation through the crimp tool was shown to agree to within $6 \%$ of the experimental results for a good crimp.

A prototype instrument for applying the technique during the wire crimping procedure was also presented along with a fundamental explanation of the technique using a wave propagation model with finite element analysis. Reproducibility between two different prototype tools was shown to be within a $95 \%$ confidence bound after calibration.

\section{Acknowledgements}

The authors would like to thank John Callahan of Lockheed-Martin Space Systems for his help in laboratory setup and data acquisition. Additionally we would like to thank John Grainger of Lockheed-Martin Space Systems and Patricia Howell of NASA Langley Research Center for their assistance in obtaining the CT images of the partially compressed crimps.

This work was performed as part of NASA's Aircraft Aging and Durability Project and was 
partially supported under Inter-Agency Agreement DTFACT-06-X-00003 with The Federal Aviation Administration.

\section{References}

1. K.E. Cramer, D.F. Perey and W.T. Yost. Proceedings of IV Pan American Conference for NDT (2007).

2. D.F. Perey, K.E. Cramer and W.T. Yost. Proceedings of the SAE Aerospace Electrical Interconnect System Symposium (2007).

3. J. D. N. Cheeke. Fundamentals and Applications of Ultrasonic Waves, pp. 411-432. CRC Press, USA, (2002).

4. R. Bracewell. The Fourier Transform and Its Applications, $3^{\text {rd }}$ ed., pp. 267-272. McGraw-Hill, New York (1999).

5. P. M. Gammell. Ultrasonics 19 (1981).

6. D. E. W. Stone and B. Clarke. J. Non-Destructive Testing (1975).

7. B. R. Jones and D. E.W. Stone. J. Non-Destructive Testing (1976).

8. B. G. Martin. NDT International 9 (1976).

9. D. K. Hsu and S. M. Nair, Review of Progress in QNDE 6B (1987).

10. J. Hashim, L. Looney and M. S. J. Hashmi. J. of Materials Processing Technology 123: 2 (2002).

11. R. Ambardar, T. Jayakumar, M. T. Muthu, and O. Prabhakar. Insight 37: 7 (1995). 


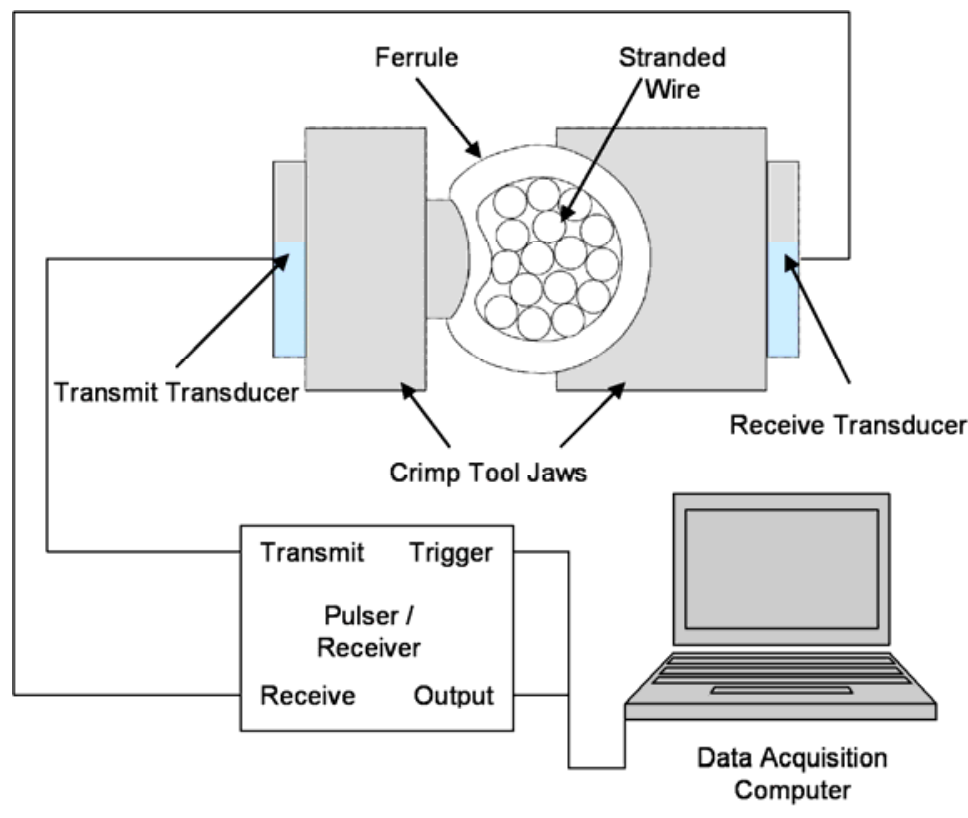

Figure 1. Block diagram of arrangement for ultrasonic interrogation of crimp connector. 


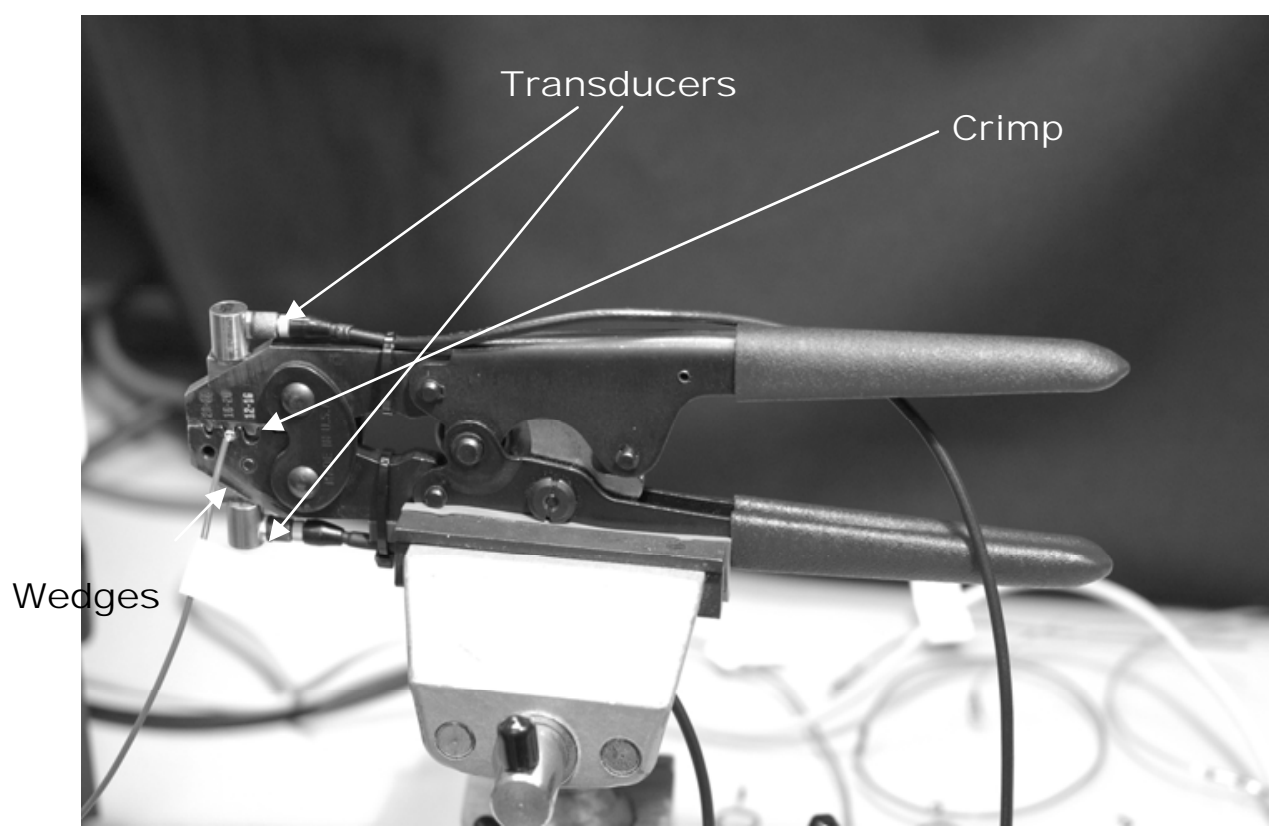

Figure 2. Photograph of the prototype instrumented crimp tool. 


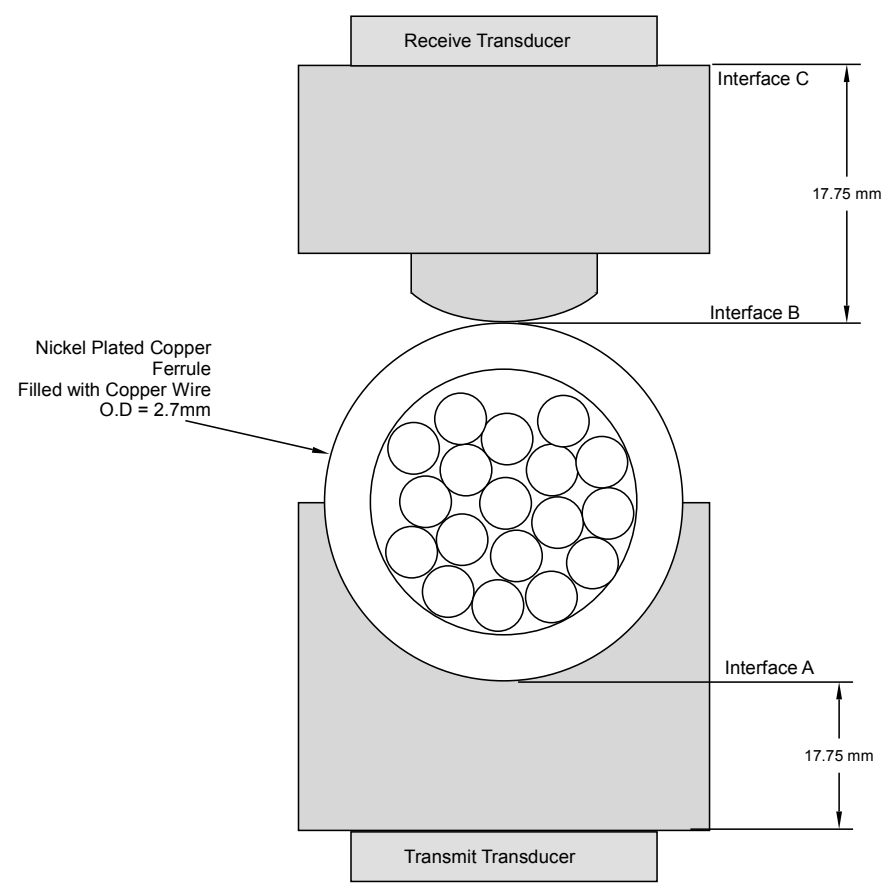

Figure 3. Drawing of electrical connector and crimp tool geometry used for wave propagation modeling (not to scale). 


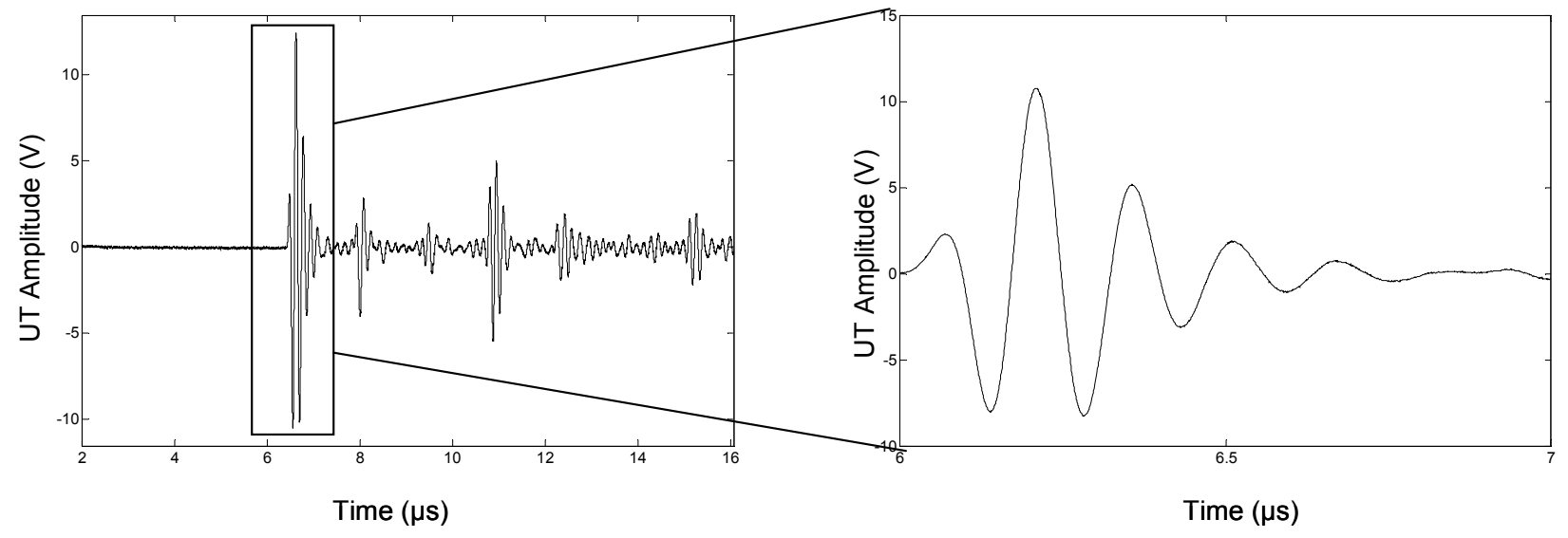

Figure 4. Ultrasonic response of a crimped electrical connector, confirming that the first arrival signal at the receive transducer occurs at approximately $6.5 \mu$ s and can therefore be modeled as compressional waves only. 


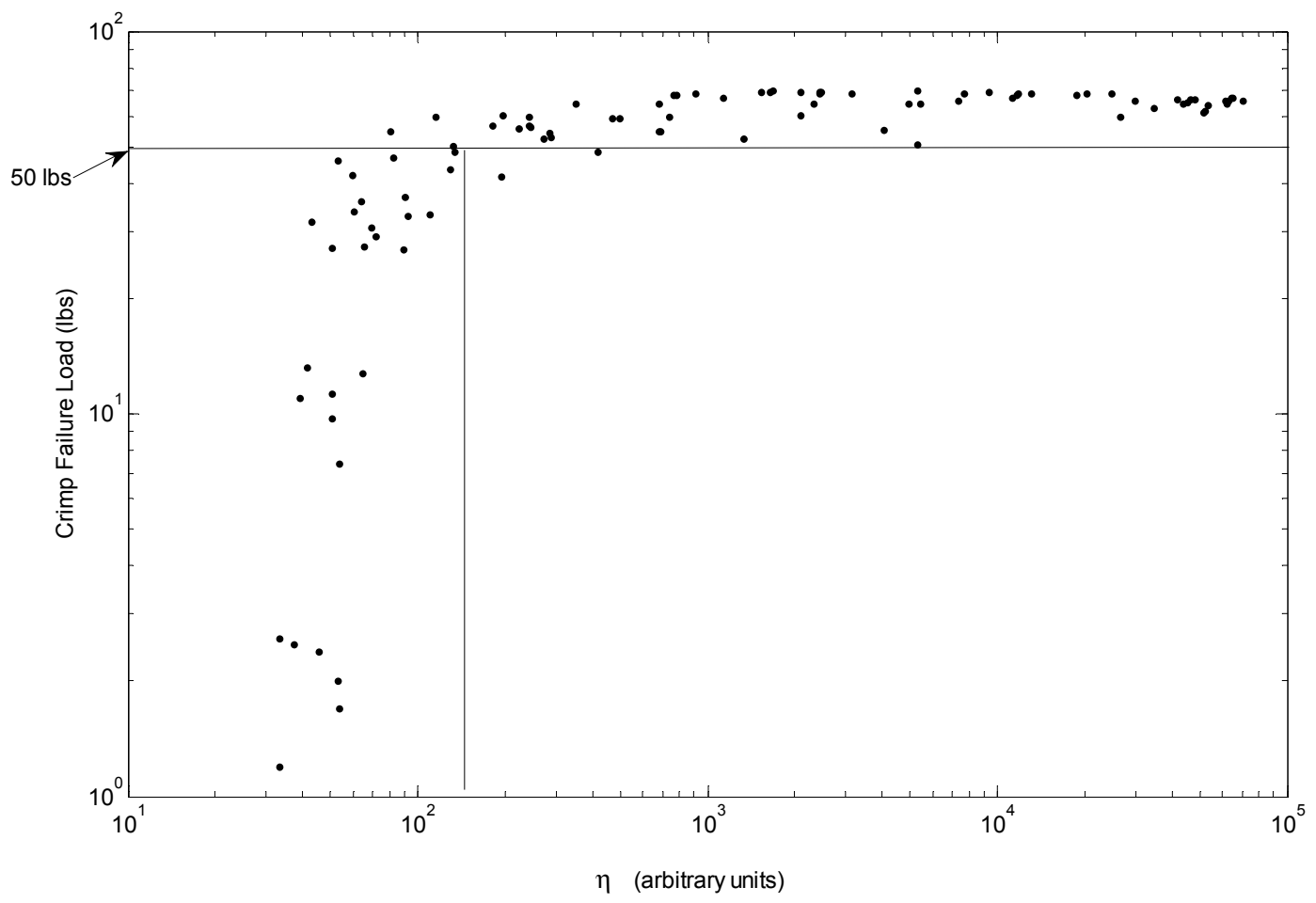

Figure 5. Plot of the crimp load at failure verses $\eta$ for the 16-gauge wire crimp specimen set. The horizontal line represents minimum load required for acceptable crimp. The vertical line represents a minimum amplitude integrated signal for an acceptable crimp for this data set. 


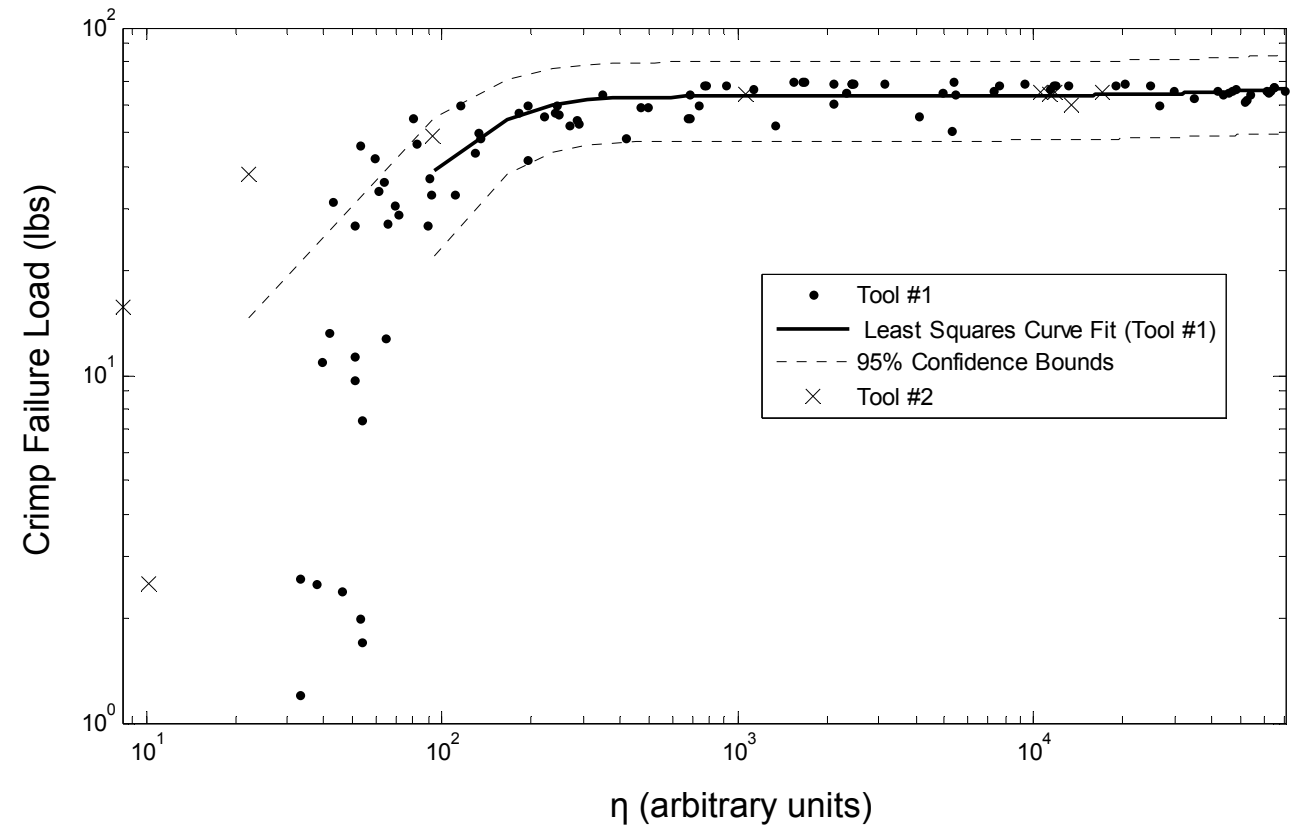

Figure 6. Comparison of the response of two different instrumented crimp tools for under-crimped conditions. 


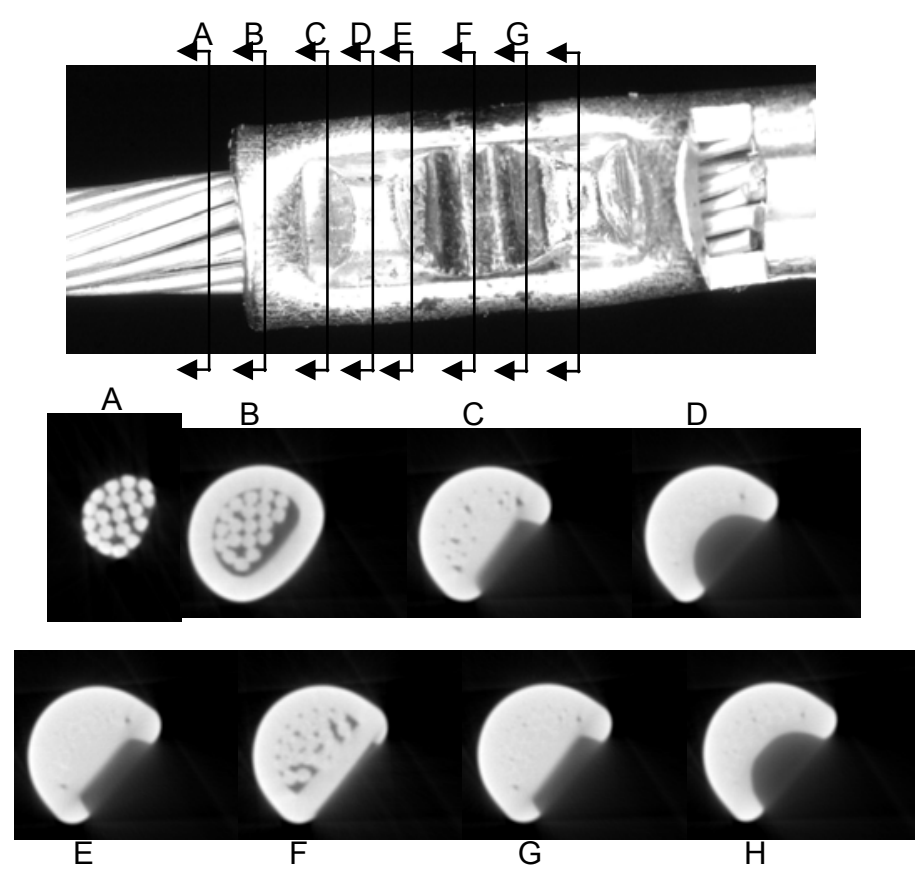

Figure 7. Photograph of fully crimped connector and computed tomography slices at eight locations across the crimped connection. 


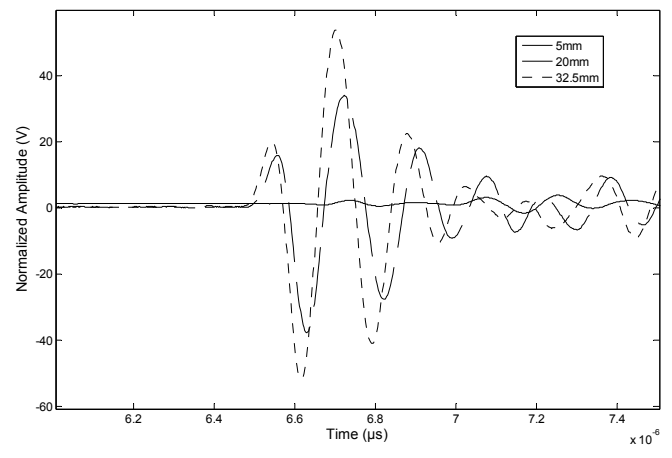

(a)

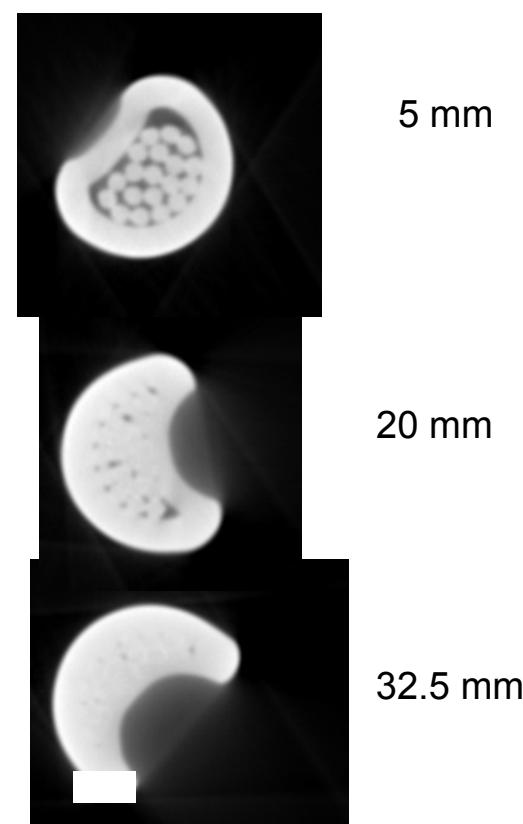

(b)

Figure 8. (a) Time windowed ultrasonic response and (b) CT images of three compression levels. 


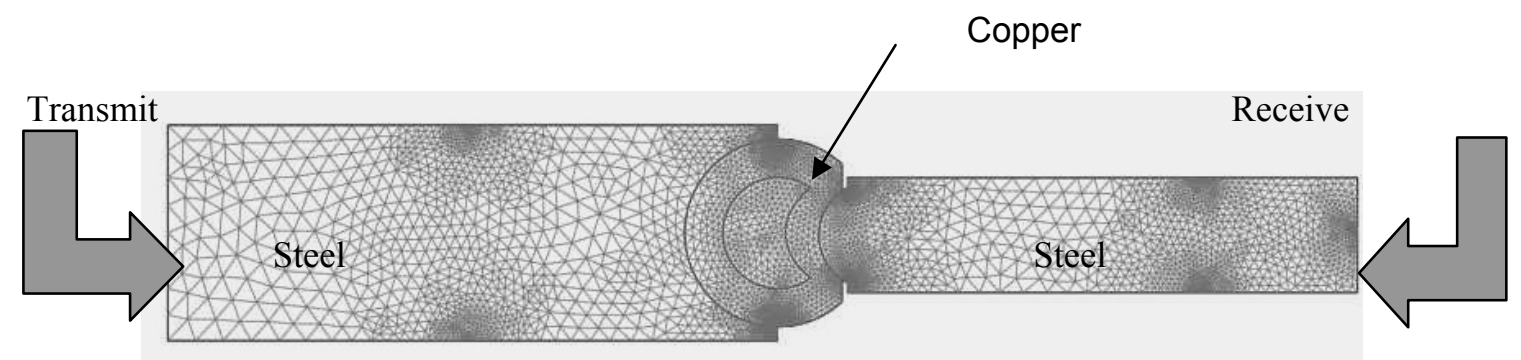

Figure 9. Finite element grid used to model the ultrasonic transmission through the crimped connector and the crimp tool jaws. 


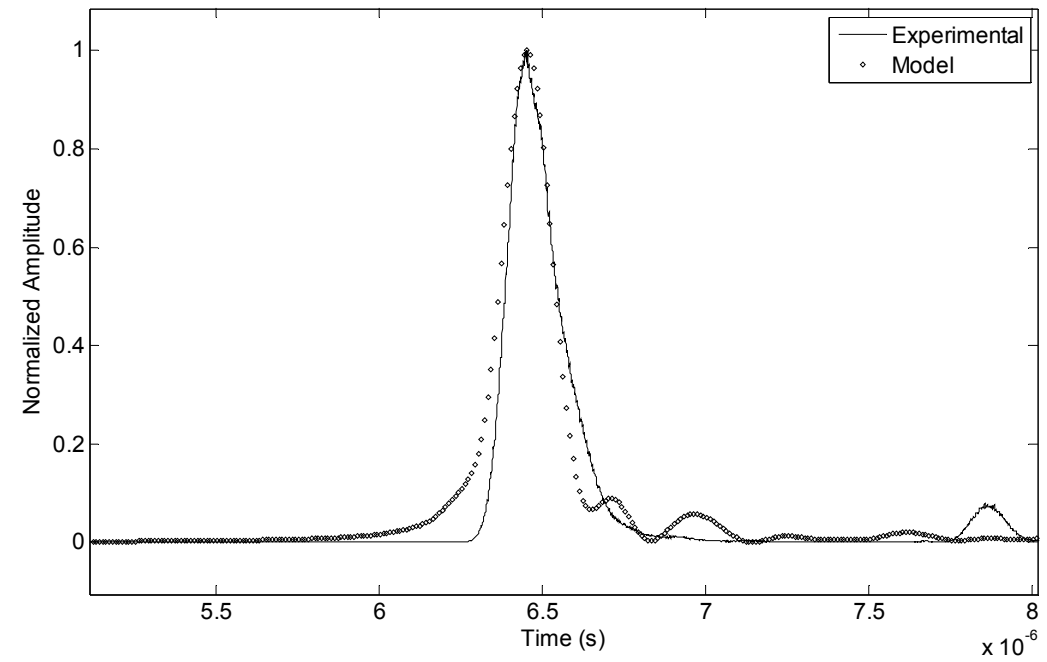

Figure 10. Comparison of the square of the modulus of the Hilbert Transforms of experimental and simulated response for a fully crimped connector. 